\title{
¿Cómo innovar en Psicobiología? Aplicación de un Ciclo de Mejora en el Aula (CIMA) para asignatura completa en el grado en Psicología
}

\author{
How to innovate in \\ Psychobiology? Application \\ of a cycle of improvement in \\ the classroom for full subject \\ in the degree of Psychology
}

ISABEL MARÍA MARTÍN MONZÓN

ORCID: https://orcid.org/0000-0002-4186-7933

Universidad de Sevilla.

Facultad de Psicología.

Departamento de Psicología Experimental.

isabelmartin@us.es

Fecha de recepción:

Fecha de aceptación:

DOI: http://dx.doi.org/10.12795/9788447221912.059

Pp.: 1349-1373 
El trabajo que se presenta corresponde a un Ciclo de Mejora (CIMA) desarrollado para la asignatura de 1o de Grado de Psicología denominada Fundamentos de Psicobiología. El modelo metodológico aplicado, las preguntas problema planteadas, la secuencia de actividades y el sistema de evaluación que se ha puesto en práctica ha puesto de manifiesto la necesidad de realizar innovación docente en el ámbito de la docencia universitaria, con el objeto de favorecer el aprendizaje de conceptos, procedimientos y actitudes de los estudiantes, generando desarrollo en sus modelos mentales. Tras el análisis de los datos de la evaluación inicial y final del CIMA se han obtenido resultados positivos, que ponen de manifiesto cómo intervenciones en el aula no convencionales favorecen no sólo la evolución en las ideas de los estudiantes sino el desarrollo profesional del docente.

Palabras Clave: Grado en Psicología, Innovación Docente, Docencia universitaria, Psicobiología, CIMA.

\section{Abstract}

The work presented corresponds to a Cycle of Improvement (CIMA) developed for the subject of 1st Degree in Psychology called Fundamentals of Psychobiology. The methodological model applied, the problem questions posed, the sequence of activities and the evaluation system that has been performed into practice has highlighted the need for teaching innovation in the field of university teaching, with the aim to improve the learning of students' concepts, procedures and attitudes, generating development in their mental models. After the analysis of the results in the initial and final evaluation of the CIMA, positive results have been obtained, which show how unconventional classroom interventions favor not only the evolution in the ideas of the students but also the professional development of the participating teacher.

Keywords:: "Degree in Psychology", "Teaching Innovation”, "University teaching", "Psychobiology", "CIMA". 


\section{Descripción del contexto}

La asignatura en la cual he aplicado el presente CIMA es "Fundamentos de Psicobiología", asignatura correspondiente al primer cuatrimestre del primer curso del grado de Psicología. Consta de 6 créditos repartidos, cada semana, en dos clases teóricas de 1 hora cada una de ellas, más 2 horas de prácticas. Es una asignatura troncal y de formación básica (obligatoria). Consta de 15 temas, agrupados en 4 unidades temáticas.

Desde el curso 2016-17 estoy realizando innovaciones respecto a mi docencia universitaria en esta asignatura (Martín-Monzón, 2017; Martín-Monzón, 2018), lo cual me ha permitido modificar progresivamente ciertos aspectos del modelo didáctico, caracterizado por el aprendizaje basado en problemas, debates entre grupos de pares (donde se ayude a generar pensamiento crítico), reflexión del estudiantado, interacción profesor-alumno, y reelaboración de los modelos mentales.

Este curso 2019-20 es el primer año en el que la asignatura ha dejado de impartirse con grupos grandes, medianos y pequeños, para pasar a una organización dividida entre grupos grandes y pequeños. Esta modificación en la estructura de la docencia me ha permitido plantearme un CIMA más integrador, que abarca por primera vez una asignatura completa, donde se continúen implantando las adaptaciones en el modelo metodológico ya aplicadas en años anteriores, y se innove en nuevos planteamientos. Así, he realizado un CIMA para asignatura completa de 30 horas presenciales distribuídas en 8 semanas lectivas.

Los principales objetivos planteados en este CIMA han sido: 1. Ampliar el mapa de contenidos del CIMA, ya que este año la innovación abarca la asignatura completa. 2. 
Consolidar los avances realizados en innovaciones previas, manteniendo los principios didácticos (Martín-Monzón, 2017, Martín-Monzón, 2018). 3. Fomentar aún más la interacción entre docente-alumno, permutando la forma tradicional de impartir docencia, por un estilo mucho más dinámico, fomentando el enfoque interactivo entre docente-alumno basado en incrementar la motivación e interés del alumnado, autoaprendizaje, debates, aprendizaje basado en problemas (mediante casos clínicos), y buscando la reelaboración de sus modelos mentales (Bain, 2007; Porlán, 2017). 4. Mejorar la evaluación del estudiante y del docente. A los estudiantes, además de evaluar su aprendizaje antes y después del CIMA, se les pidió realizar un portafolio, donde resumieron las ideas principales trabajadas en las sesiones de clase. Asimismo, se introduce la novedad de valorar la práctica docente por parte de los estudiantes a través de un cuestionario, y por parte del docente a través de su propio diario personal.

\section{Mapa de contenidos y problemas}

El mapa de contenidos se basó en las unidades del actual programa de la asignatura, las cuales comprenden 15 temas de Fundamentos Psicobiológicos, distribuídos en 4 bloques de unidades. En este CIMA se plantean contenidos conceptuales (relacionados con la teoría de la asignatura), aspectos procedimentales (uso y manejo del microscopio óptico, descripción de procedimientos histológicos y de manejo de sujetos experimentales en casos de lesiones) y actitudinales (valores de investigación).

Para poder organizar dicho contenido, el proceso de reflexión sobre la relevancia de los mismos fue clave. Resultó interesante el trabajo de seleccionar los contenidos organizadores y las preguntas clave en una asignatura tan densa y de dificil comprensión como ésta, donde todo está relacionado. A veces es mejor reducir parte de los 
contenidos y darle prioridad a la reflexión e implicación del alumnado.

Teniendo en cuenta lo anterior se distinguen los siguientes grandes bloques de contenidos/problemas, siendo dos semanas la duración de cada uno de ellos:

Unidad I. ¿Qué es la Psicobiología? ¿Qué métodos emplea esta disciplina? [Concepto de Psicobiología y Métodos de Investigación]

Unidad II. ¿Cómo explicar la conducta a partir de la herencia genética? [Fundamentos de Genética de la Conducta y Evolución]

Unidad III. ¿Cómo se genera, transmiten e integra el impulso nervioso? [Conducción, transmisión y la integración de señales neurales]

Unidad IV. ¿Cuál es la organización general del Sistema Nervioso Central y la funcionalidad de sus subunidades básicas? [Neuroanatomía funcional].

A continuación, en la Figura 1 se muestra el mapa de contenidos, con las preguntas claves que han guiado el CIMA.

Jornadas de Formación e Innovación Docente del Profesorado | № 2 (2019) Esta obra se distribuye con la licencia Creative Commons 


\section{ISABEL MARÍA MARTÍN MONZÓN}
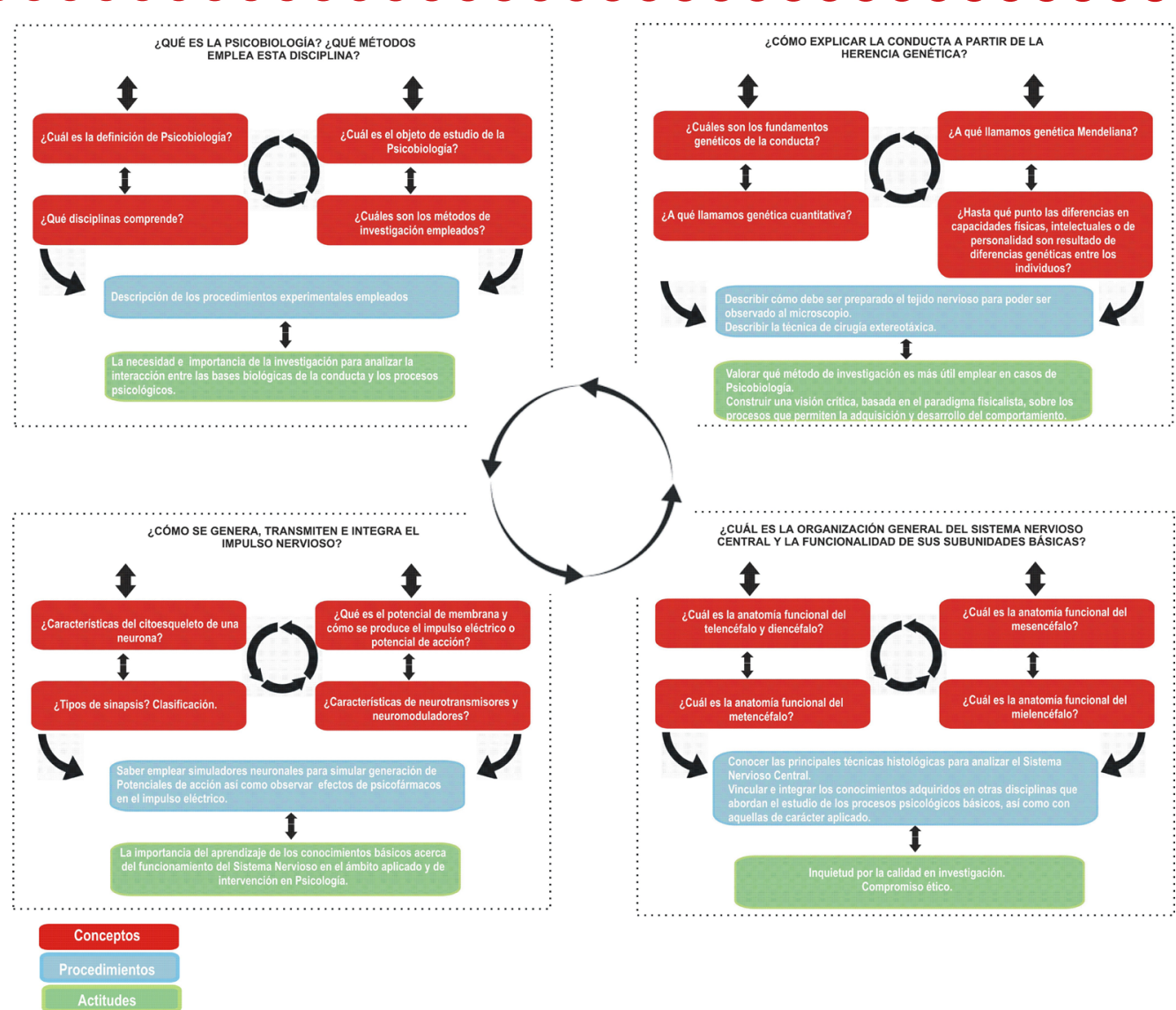

Figura 1. Mapa de contenidos a desarrollar y relación con las preguntas problema.

Jornadas de Formación e Innovación Docente del Profesorado | № 2 (2019)

(c) (i) $\odot$ Esta obra se distribuye con la licencia Creative Commons 


\section{Modelo metodológico posible y secuencia de actividades}

Para el desarrollo de este CIMA de asignatura completa y teniendo en cuenta los avances establecidos ya en los dos años previos de trabajo en innovación en la asignatura, se estableció un modelo metodológico que partiendo de un problema general (¿Cuáles son las bases biológicas de la conducta?), y varios específicos (véase Figura 1), se desarrolle una secuencia de actividades que permita el desarrollo progresivo de las ideas de los estudiantes sobre el mismo, hasta llegar a una conclusión final. De esta manera, el modelo metodológico planteado permite que los estudiantes reflexionen sobre los problemas genéricos o específicos planteados, ayudándoles a elaborar diferentes puntos de vista a partir de las cuestiones o debates a resolver en clase.

En este modelo metodológico diseñado, el alumno es el protagonista. Se plantea un modelo de metodología investigativa, donde se busca fomentar en el alumnado la motivación, la curiosidad y la atención, para conseguir un aprendizaje duradero en el tiempo (Mora, 2017). Se emplearon diferentes estrategias (Finkel, 2008) para poder mantener el dinamismo en la clase (ej: desplazar a la profesora del centro del aula, dar clase con la boca cerrada...), intentando que los alumnos fueran activos durante la clase, lo cual requiere de un alto grado de compromiso y dedicación y responsabilidad por parte de los mismos.

Especificamente, el modelo metodológico abarcaba diferentes fases (distintas según fuera clase de Grupo Grande o Grupo Pequeño, Figura 2). En general, se aplicó empleando un formato donde predominaron los debates derivados de problemas o casos que fueron subidos a la plataforma de enseñanza virtual de la asignatura (en adelante EV) al menos con una semana de anterioridad. A dichos debates, en primer lugar realizados por parejas, y 
posteriormente en pequeños grupos de alumnos, les sucedió una fase de contraste, que sustituyendo a la tradicional fase de explicación teórica, consistía en la exposición docente de argumentos teóricos y empíricos para resolver las dudas y cuestionar las concepciones de los estudiantes. De esta forma, el fin docente no es únicamente generar conocimiento en los estudiantes (punto de vista tradicional), sino que se consigue algo más profundo, y es hacer reflexionar a los alumnos y cuestionar sus puntos de vista, para ayudarles a elaborar otros mejores.

La interacción entre lo impartido y trabajado en clases de grupo grande y clases de práctica en laboratorio se mantuvo a lo largo de todo el CIMA, de manera que al finalizar cada sesión, la docente realizaba un resumen de las ideas principales, relacionando lo observado y trabajado en dicha sesión con lo trabajo en grupo grande o grupo pequeño, de manera que se establecía una continuidad entre todas las sesiones de la asignatura.

Jornadas de Formación e Innovación Docente del Profesorado | № 2 (2019) Esta obra se distribuye con la licencia Creative Commons 
NoP. Actividad no presencial antes de la sesión de grupo grande. Alumnos trabajan con cuestiones subidas a la plataforma EV.

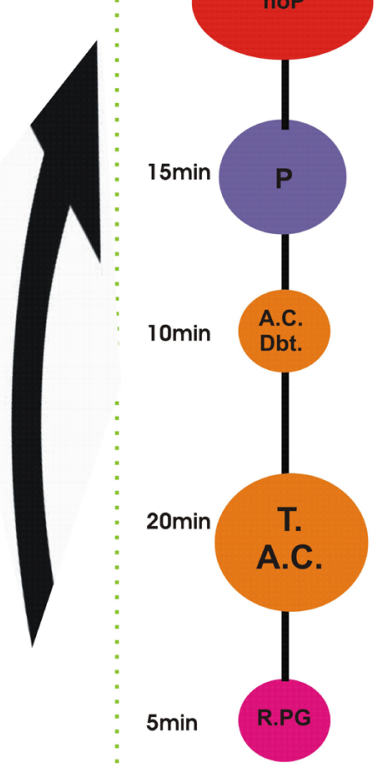

$\checkmark$

P. Contraste de resolución de cuestiones en clase por parejas. Los alumnos deben preparar dudas y preguntas a la profesora por escrito para tenerlas en cuenta en la siguiente fase.

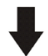

A.C. Dbt. Los alumnos en grupos pequeños, deben continuar preparando dudas y preguntas a la profesora por escrito. Se establecen debates y acuerdos entre los alumnos para identificar sus principales dudas del tema, con el objeto de ser aclaradas por la prof en la siguiente fase. 1

T. A.C. Actividad de contraste, donde más que transmitir directamente la teoría, la profesora expone argumentos y datos que resuelvan o cuestionen las ideas de los estudiantes.

R. PG. Breve resumen de clase y preguntas "gancho" por parte de la docente, enlazando lo impartido en esta hora con los contenidos ya impartidos y con los que se impartirán en la siguiente clase.
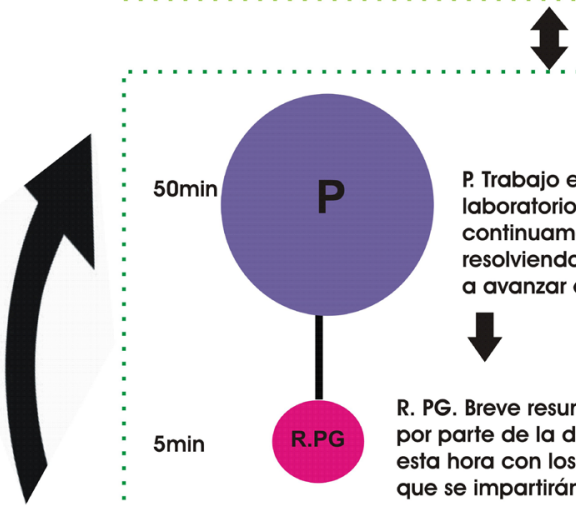

Figura 2. Modelo metodológico posible. El interlineado verde corresponde a las horas de grupo grande; el interlineado azul a las horas de grupo pequeño.

Siguiendo el modelo metodológico planteado, se expone en la Tabla 1 la secuencia de actividades programada y desarrollada. 
Tabla 1. Cronograma de actividades desarrollado en el CIMA de Fundamentos de Psicobiología.

\begin{tabular}{|c|c|}
\hline $\begin{array}{l}\text { Semana y } \\
\text { Contenido }\end{array}$ & Actividades de contraste \\
\hline $\begin{array}{l}\text { 1. Semana del } 23 \\
\text { de septiembre } \\
\text { y semana del } \mathbf{3 0} \\
\text { de septiembre. } \\
\text { Concepto de } \\
\text { Psicobiología } \\
\text { y métodos de } \\
\text { investigación. }\end{array}$ & $\begin{array}{l}\text { Grupo grande: } \\
\text { - Cuestionario inicial de la asignatura (valoración } \\
\text { estudiantes). } \\
\text { - Cuestión-problema: ¿Cuál es la definición de Psico- } \\
\text { biología?. Actividad planteada: Distinguir las dife- } \\
\text { rencias y semejanzas entre las diferentes ramas de } \\
\text { la Neurociencia e identificar lo que caracteriza a la } \\
\text { Psicobiología. } \\
\text { Cuestión-problema: ¿Cuál es el objeto de estudio de } \\
\text { la Psicobiología?. Actividad planteada: Analizando di- } \\
\text { ferentes textos que la docente haya dejado en la EV, } \\
\text { discriminar el objeto de estudio de la disciplina Psi- } \\
\text { cobiológica. Se puede plantear un debate en clase } \\
\text { para trabajar esta idea. } \\
\text { Cuestión-problema: ¿Qué disciplinas comprende?. } \\
\text { Actividad planteada: Analizando diferentes textos } \\
\text { que la docente haya dejado en la EV, discriminar el } \\
\text { objeto de estudio de la disciplina Psicobiológica. } \\
\text { Se puede plantear un debate en clase para trabajar } \\
\text { esta idea. } \\
\text { Cuestión-problema: ¿Cuáles son los métodos de in- } \\
\text { vestigación?. Actividad planteada: Tras la explicación } \\
\text { teórica de la profesora, se plantea debate para tratar } \\
\text { de diferenciar entre métodos experimentales y no } \\
\text { experimentales, invasivos y no invasivos. }\end{array}$ \\
\hline
\end{tabular}

Jornadas de Formación e Innovación Docente del Profesorado | № 2 (2019) Esta obra se distribuye con la licencia Creative Commons Reconocimiento-NoComercial-SinObraDerivada 4.0 Internacional (CC BY-NC-ND 4.0.) 


\section{Semana del $\quad$ Grupo grande:}

7 de octubre $y$ semana del 14 de octubre.

Fundamentos de genéticos de la conducta y evolución.

- Cuestión-problema: ¿Cuáles son los fundamentos genéticos de la conducta?. Actividad planteada: debate de caso clínico de hidrocefalia.

- Cuestión-problema: ¿A qué llamamos genética Mendeliana?. Actividad planteada: realización de problemas genéticos, donde los alumnos puedan trabajar en pares, para posteriormente debatir los resultados con otros compañeros y profesora.

- Cuestión-problema: ¿A qué llamamos genética cuantitativa?. Actividad planteada: realización de problemas genéticos, donde los alumnos puedan trabajar en pares, para posteriormente debatir los resultados con otros compañeros y profesora.

- Cuestión-problema: ¿Hasta qué punto las diferencias en capacidades físicas, intelectuales o de personalidad son resultado de diferencias genéticas entre los individuos? Actividad planteada: debate de casos clínicos con alteraciones genómicas y afectación conductual. 


\section{Semana del \\ Grupo grande:} 21 de octubre $y$ semana del 28 de octubre.

Conducción, transmisión e integración de señales.
- Cuestión-problema: ¿Características del citoesqueleto de una neurona?. Actividad planteada: analizar a través de microscopía óptica y describir la citoarquitectura neuronal empleando la tinción de Golgi. Trabajo en pares.

- Cuestión-problema: ¿Qué es el potencial de membrana y cómo se produce el impulso eléctrico o potencial de acción?. Actividad planteada: emplear simulación neuronal (cd del manual de laboratorio de prácticas) para resolver diferentes casos planteados con alteración de canales de $\mathrm{Na}+\mathrm{y}$ de $\mathrm{K}+$ en la membrana neuronal.

- Cuestión-problema: ¿Tipos de sinapsis? Clasificación. Actividad planteada: tras la lectura de documentos dejados en EV, los alumnos deben debatir en pares y posteriormente en grupos más grandes de alumnos los tipos de sinapsis (químicas, eléctricas) y su funcionalidad, así como clasificación. Posteriormente la profesora explicaría en detalle estas cualidades.

- Cuestión-problema: ¿Características de neurotransmisores y neuromoduladores?. Actividad planteada: lectura y debate de casos clínicos con patologías tales como trastornos de ansiedad, trastornos depresivos u esquizofrenia. Identificación de correspondencias entre patologías y tipos de neurotransmisores o neuromoduladores probablemente alterados así como sus receptores.

\section{Grupo pequeño:}

- Cuestión-problema: ¿Cómo discriminar tipos celulares según el citoesqueleto?. Actividad planteada: empleando el microscopio óptico, analizar la citoarquitectura neuronal para identificar tipos celulares en las 3 capas del cerebelo, así como localizar cada una de las estructuras básicas de la neurona: soma, axón y dendritas de cada uno de los tipos celulares. 


\section{Semana del 4 de noviembre y del 11 de noviembre} Neuroanatomia funcional

\section{Grupo grande:}

- Cuestión-problema: ¿Cuál es la anatomía funcional del telencéfalo y diencéfalo?. Actividad planteada: explicaciones teóricas, análisis de casos clínicos, uso de maqueta de neuroanatomía en grupos de alumnos y debate.

- Cuestión-problema: ¿Cuál es la anatomía funcional del mesencéfalo?. Actividad planteada: análisis de imágenes de neuroanatomía a partir de manuales de referencia de la asignatura, caso clínico y software Sylvius. Explicaciones teóricas y trabajo práctico en laboratorio con maquetas cerebrales.

- Cuestión-problema: ¿Cuál es la anatomía funcional del metencéfalo?. Actividad planteada: análisis de imágenes de neuroanatomía a partir de manuales de referencia de la asignatura, empleo de maqueta, caso clínico y software Sylvius. Explicaciones teóricas y trabajo práctico en laboratorio con maquetas cerebrales.

- Cuestión-problema: ¿Cuál es la anatomía funcional del mielencéfalo?. Actividad planteada: análisis de casos clínicos, uso de maqueta de neuroanatomía en grupos de alumnos y debate. Explicaciones teóricas y trabajo práctico en laboratorio con maquetas cerebrales.

- Cuestionario final de la asignatura (evaluación del alumnado y de la docente)

\section{Grupo pequeño:}

- Cuestión-problema: ¿Qué estructuras del prosencéfalo se pueden observar con una sección sagital medial?. Actividad planteada: empleando maquetas cerebrales trabajar en la localización de estructuras mediante diferentes planos de corte: sagital, horizontal y coronal. 


\section{Cuestionario inicial-final de seguimiento de la evolución de las ideas de los estudiantes}

Para poder valorar la evolución en el aprendizaje de los estudiantes, se desarrolló un cuestionario inicial y final que permitió a la docente identificar los modelos mentales que predominaban en la clase, así como su evolución. Se intentó generar un cuestionario que representara las principales cuestiones que un futuro psicólogo debe plantearse sobre las bases biológicas de la conducta. Cada una de las cuestiones planteadas tienen una relación directa con cada uno de los problemas que se han trabajado en la secuencia de actividades (Tabla 1). Como se puede ver a continuación, las cuestiones 1, 3 y 4 están relacionadas intrínsecamente con lo planteado en las semanas dedicadas al "Concepto de Psicobiología y métodos de investigación", la cuestión 2 con las actividades de contraste de las sesiones dedicadas a "Fundamentos genéticos de la conducta y evolución", las cuestiones 5 y 6 con los problemas planteados en "Conducción, transmisión e integración de señales", y finalmente las cuestiones 7 y 8 con lo trabajado en las semanas dedicadas a "Neuroanatomía funcional". El instrumento de recogida de ideas ha sido el siguiente:

Alumno/a:

Por favor, responda a las siguientes cuestiones:

1. ¿Qué es la Psicobiología y cuál es su objeto de estudio?

2. ¿Cuáles son los factores epigenéticos que pueden influir en la conducta de un individuo?

3. ¿Cuál es el método de investigación empleado en Psicobiología?

4. Identifique técnicas para el estudio del cerebro humano "in vivo".

5. Defina el potencial de acción así como sus principales fases.

6. Identifique las principales diferencias entre neurotransmisores y neuromoduladores. 
7. ¿Cuáles son las principales subdivisiones del Sistema Nervioso Central (SNC)?

8. ¿Cuáles son los mecanismos y sistemas que contribuyen a la protección y funcionamiento del SN?

\section{Diario de las sesiones}

El CIMA comenzó a aplicarse el primer día de clase lectiva del cuatrimestre, de manera que se le explicó al alumnado la metodología de tipo investigativo que se iba a aplicar, y que se pretendía realizar una evaluación inicial y final de su evolución a lo largo de las semanas de clase. Una vez realizado el cuestionario, pregunté en voz alta el parecer de los alumnos con respecto a las cuestiones. En general todos los alumnos indicaron que no tenían mucho conocimiento de lo que se les preguntaba (puesto que para acceder a Psicología no es necesario proceder desde el itinerario de Ciencias), pero también había alumnos que sí conocían algo la materia, y lo que necesitaban era profundizar en la misma, lo cual era el objetivo fundamental de nuestra asignatura. Este primer día fue muy interesante desde el punto de vista docente, puesto que me permitió comenzar las clases el día siguiente con explicaciones más básicas que las que normalmente empleo, y por tanto adaptándome a los perfiles mentales que este año tenía en clase. Todo ello lo dejé reflejado en el "diario del profesor", que comencé tras esta primera sesión y lo he continuado hasta el final del CIMA. Las semanas de docencia transcurrieron sin grandes incidencias en la asignatura, de manera que cada semana se les subía a la plataforma virtual EV las cuestiones que debían trabajar previamente en casa para poder debatirlas en clase. En este sentido, he de decir que durante dos semanas en octubre (correspondientes a sesiones de "Fundamentos genéticos de la conducta y evolución"), los alumnos no pudieron realizar las lecturas previamente (por falta de tiempo debido a exámenes de otras asignaturas), y ello conllevó a que la 
metodología planteada perdiera dinamismo. No obstante, el clima que se generó en clase desde las primeras sesiones, me permitió el diálogo continuo con los alumnos, y valorar algún cambio en la asignatura cuando fue necesario. Tras conversación con los alumnos, éstos me indicaron expresamente que querían seguir realizando las cuestiones semanales, participando de las actividades de contraste como hasta la fecha, ya que era una manera diferente de aprender Psicobiología, que les motivaba más que con otro tipo de enseñanza más tradicional en otras asignaturas. Con estos diálogos, los alumnos entendían que sus aportaciones no eran valoradas o cuestionadas, sino reforzadas o incitadas al replanteamiento. Además, sabían que sin participar activamente en cada unidad temática, no podrían entregar los portfolios que aunque no eran obligatorios, sí eran compensados con el 5\% de la calificación final de la asignatura. En general, el clima en clase fue positivo, de forma que fue un factor clave para mantener la metodología propuesta. Tras esta incidencia, se continuó aplicando el CIMA, impartiendo docencia para favorecer la adquisición del contenido tanto conceptual, como procedimental y actitudinal de una manera distinta a la tradicional.

Transcurridas las cuatro primeras semanas lectivas, comenzaron las prácticas de grupo pequeño de la asignatura, de manera que la secuencia de actividades planteada en la semana se diversificó y potenció, permitiendo trabajar directamente con los alumnos sobre aspectos procedimentales y actitudinales en Psicobiología. Estas clases fueron muy dinámicas, de manera que trabajaban por pares con un microscopio y/o maqueta cerebral.

El último día del CIMA, tuvo lugar la aplicación del cuestionario final. Los alumnos que asistieron a clase fueron un $18 \%$ menos que el primer día de clase, puesto que a lo largo del cuatrimestre ha ido disminuyendo levemente el número de asistentes a clases. Este mismo día, también se 
les entregó un cuestionario sobre valoración docente que permitió obtener retroalimentación por mi parte para así mejorar la práctica docente en futuras sesiones. La realización del cuestionario inicial-final ha resultado ser un factor decisivo para poder valorar cómo influye la práctica docente en los modelos mentales. Es de recalcar, que si bien muchos partían del nivel D ó C (las respuestas fueron clasificadas en 4 niveles, siendo $D$ el más básico y el $A$ el más complejo y completo en la escala de aprendizaje), el nivel $D$ desapareció en la evaluación final. Con respecto al nivel A, de la misma manera pero a la inversa, éste no se presentaba en los inicios de las respuestas, y finalmente fue el más presente en todas las cuestiones evaluadas.

\section{Evaluación del aprendizaje de los estudiantes y valoración de la docencia}

La evaluación de los modelos mentales de los estudiantes se ha realizado a través la comparación de sus respuestas en los cuestionarios inicial-final del presente CIMA, analizados a través de escaleras de aprendizaje. En la mayoría de las cuestiones, se ha producido una evolución de la mayor parte de los alumnos encuestados. Se ha observado una gran heterogeneidad entre las respuestas de los alumnos. De 50 alumnos (100\%) que han participado en esta evaluación, un 20\% no habían recibido formación en Biología en el curso previo (en 2o Bachiller), todos ellos provenientes de itinerario no científico, mientras que el 80\% restante sí (15\% alumnos repetidores de Grado, 65\% procedentes de 2o Bachiller u otros Grados de Ciencias). Se observó que preguntas relacionadas intrínsecamente con la definición de la asignatura o sus aspectos teóricos más básicos, no formaban parte de su conocimiento básico (1, 2 y 3), mientras que sí respondieron algunos alumnos a preguntas más biológicas (que no psicobiológicas), las cuales podrían haberlas aprendido parcialmente en cursos anteriores $(4, y 5)$. Las preguntas más especializadas fueron 
respondidas por algunos alumnos pero no supieron responderlas con el nivel apropiado (6, 7 y 8). La evaluación inicial-final resultó muy interesante no sólo para la docente sino también para los alumnos, puesto que les sirvió para darse cuenta de lo aprendido.

A continuación, se presentan en formato de escaleras de aprendizaje los resultados más relevantes obtenidos en las cuestiones: cuestión 1 (Tabla 2), cuestión 2 (Tabla 3), cuestión 5 (Tabla 4) y cuestión 8 (Tabla 5). Por falta de espacio disponible no se describen el resto de resultados.

Tabla 2. Progresión de aprendizaje en la cuestión 1 “Qué es la Psicobiología y cuál es su objeto de estudio?"

\section{CUESTIONARIO INICIAL}

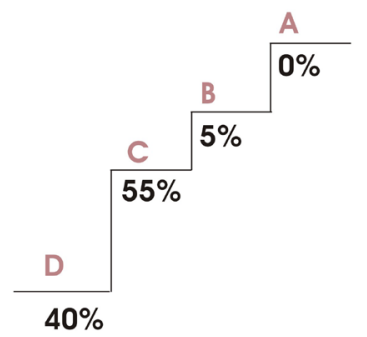

\section{CUESTIONARIO FINAL}

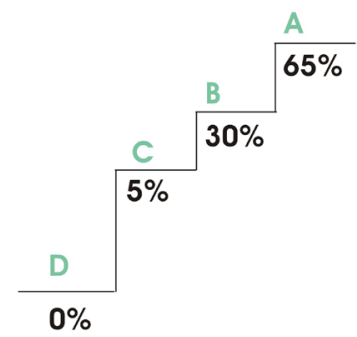

\section{Cuestión-problema.¿Qué es la Psicobiología y cuál es su objeto de estudio?}

Modelos mentales: MODELO A. Indica correctamente la definición más aceptada en el marco científico de lo que es la Psicobiología. MODELO B. Identifica correctamente el objeto de estudio pero no su marco teórico. MODELO C. Confunde el objeto de estudio y el marco téorico. MODELO D. No sabe/no contesta. .

Jornadas de Formación e Innovación Docente del Profesorado | № 2 (2019) Esta obra se distribuye con la licencia Creative Commons Reconocimiento-NoComercial-SinObraDerivada 
Tabla 3. Progresión de aprendizaje en la cuestión 2 ¿Cuáles son los factores epigenéticos que pueden influir en la conducta de un individuo?"
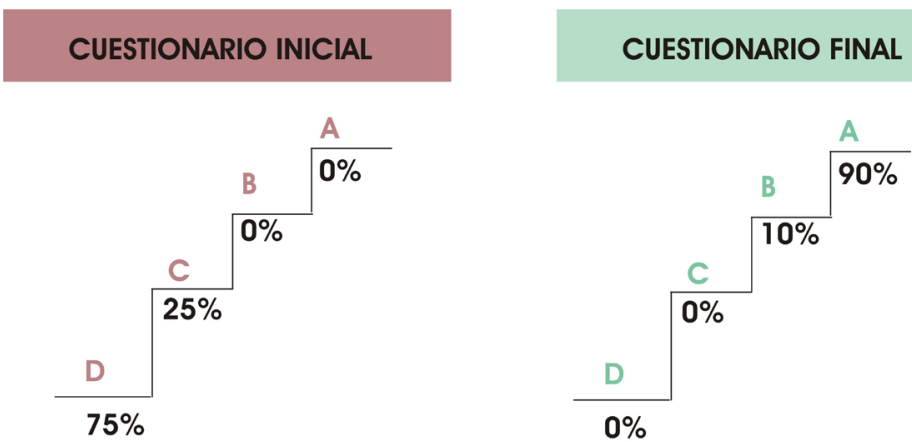

Cuestión-problema. Defina el potencial de acción así como sus principales fases. Modelos mentales: MODELO A. Define el potencial de acción correctamente e identifica cada una de sus fases de acuerdo a los cambios en la permeabilidad de la membrana celular a iones específicos (en concreto, sodio y potasio). MODELO B. Define el potencial de acción, e identifica parcialmente sus fases. MODELO C. Define el potencial de acción, pero no identifica sus fases. MODELO D. No sabe/no contesta.

Tabla 4. Progresión de aprendizaje en la cuestión 5 "Defina el potencial de acción así como sus principales fases"
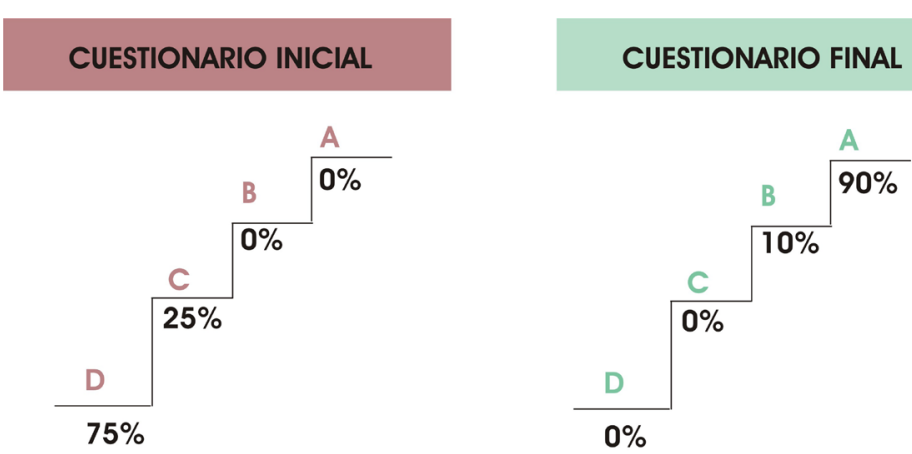

Cuestión-problema. Defina el potencial de acción así como sus principales fases. Modelos mentales: MODELO A. Define el potencial de acción correctamente e identifica cada una de sus fases de acuerdo a los cambios en la permeabilidad de la membrana celular a iones específicos (en concreto, sodio y potasio). MODELO B. Define el potencial de acción, e identifica parcialmente sus fases. MODELO C. Define el potencial de acción, pero no identifica sus fases. MODELO D. No sabe/no contesta.

Jornadas de Formación e Innovación Docente del Profesorado | № 2 (2019) Esta obra se distribuye con la licencia Creative Commons Reconocimiento-NoComercial-SinObraDerivada Internacional (CC BY-NC-ND 4.0.) 
Tabla 5. Progresión de aprendizaje en la cuestión 8 "Cuáles son los mecanismos y sistemas que contribuyen a la protección y funcionamiento del SN?"

\section{CUESTIONARIO INICIAL}

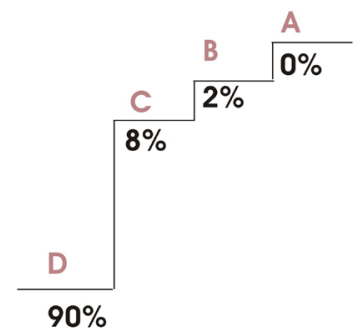

\section{CUESTIONARIO FINAL}

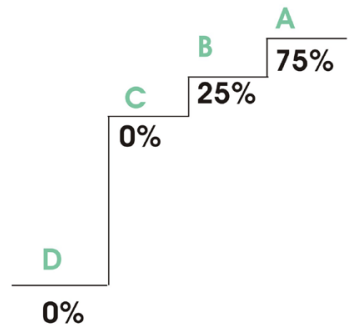

Cuestión-problema.¿Cuáles son los mecanismos y sistemas que contribuyen a la protección y funcionamiento del Sistema Nervioso Central?

Modelos mentales: MODELO A. Indica correctamente cuáles son los mecanismos y sistemas de protección del Sistema Nervioso Central. MODELO B. Identifica únicamente a las meninges y no al sistema ventricular. MODELO C. Confunde los mecanismos de protección. MODELO D. No sabe/no contesta..

Como se puede observar en las tablas, se produjo un cambio de aprendizaje entre el comienzo y final del CIMA, puesto que los alumnos respondieron de manera diferente a las cuatro cuestiones que aquí se presentan. Así, respecto a los resultados obtenidos, podemos observar cómo se produjo un cambio en los modelos mentales de los estudiantes. Si bien al comienzo del CIMA los modelos mentales de los alumnos se encontraban en los escalones más bajos (C, D), tras completar el ciclo de mejora ascendieron en todos los casos, llegando a alcanzar el modelo A el 70\% o más de los estudiantes en todos los casos (en la cuestión 2, 80\% de los estudiantes alcanzaron el modelo A; en la cuestión 5, 90\% de los estudiantes alcanzaron el modelo A; en la cuestión $8,75 \%$ de los estudiantes alcanzaron el modelo A), a excepción de la cuestión 1. Este dato es relevante, proporcionándonos la información de que se debe mejorar la docencia relativa a las sesiones donde se impartió la definición de Psicobiología y la distinción de esta disciplina científica con respecto a otras en futuros cursos. 
Por otro lado, respecto a la evaluación de la docencia, al finalizar el CIMA, se solicitó a cada alumno su opinión sobre la metodología aplicada a través de las siguientes cuestiones clave: 1. ¿Considera que ha aprendido de una forma más duradera con este tipo de metodología que con la tradicional?; 2. ¿Se encuentra más motivado con este tipo de dinámica?. Los resultados indican que el $70 \%$ de los estudiantes encuestados consideran que esta metodología es más efectiva y duradera que la tradicional, mientras que el $80 \%$ de los estudiantes indican que se motivan más con este tipo de dinámica. Estos resultados han sido muy positivos, y ayudan a mejorar e idear nuevas dinámicas de aprendizaje en el aula para seguir fomentando el aprendizaje a largo plazo.

\section{Evaluación del CIMA}

La evaluación del CIMA ha sido muy positiva, obteniendo resultados que apoyan al docente a continuar en cursos venideros con una metodología docente donde se fomenta el aprendizaje dinámico el alumnado, y se innova no sólo en la organización de los contenidos, sino en la forma de enseñar procedimientos y actitudes al grupo de alumnos.

El objetivo principal del CIMA se ha cumplido: se ha puesto en práctica un modelo de innovación docente basado en actividades de contraste, secuencia de actividades, dinámicas de aprendizaje, interacción alumno-profesor continua, y aplicación de un sistema de evaluación inicial-final, así como valoración docente.

Al reflexionar sobre aspectos relativos al contenido de las cuestiones-problema propuestas, la organización, la metodología diseñada, y la evaluación, se pueden subrayar algunos avances que ha supuesto este CIMA y otros aspectos que deberían mejorarse, destacando: 
Avances realizados:

- La realización del primer CIMA en una asignatura de Psicobiología, asignatura generalmente considerada compleja por los estudiantes. La secuencia de actividades propuestas ha ayudado a los estudiantes a mantener la motivación adecuada para avanzar en la asignatura.

- El mapa de contenidos propuesto y trabajado recoge los elementos esenciales para el problema general: "¿Cuáles son las bases biológicas de la conducta?".

- El modelo metodológico presentado se ha desarrollado como se diseñó, con la secuencia de actividades propuestas.

- La secuencia de actividades planteada (a excepción de las actividades de "Definición de Psicobiología"). En general, cada actividad del tema ha estado directamente relacionada con la anterior y la posterior (tanto en grupo grande como en pequeño), lo que ha permitido dar continuidad a su desarrollo y al aprendizaje final. Asimismo, al inicio de cada tema, las actividades estaban directamente relacionadas con el tema anterior, lo que ha permitido el repaso periódico y acumulativo de la materia.

- La escalera de aprendizaje aportada en cada cuestión del cuestionario inicial-final, ha permitido, a la docente y al estudiante, conocer la progresión del aprendizaje, así como los contenidos o actividades que presentan dificultades.

- El diario docente. Éste ha servido para poder reflexionar sesión a sesión sobre la práctica docente, para poder realizar posibles mejoras en la siguiente clase, de manera que es un proceso de evaluación continua.

- El cuestionario final de satisfacción sobre el proceso de enseñanza y aprendizaje ha ayudado a la docente a poder conocer las opiniones directas del alumnado. 
Aspectos a mejorar:

- Es preciso modificar las actividades vinculadas a las primeras sesiones del CIMA, ya que es donde se ha obtenido un porcentaje menor en el modelo mental A. Debo mejorar e idear sesiones más motivantes y atractivas para el alumnado, con el objetivo de que aprendan correctamente la base de esta disciplina. Una manera podría ser realizar debates con aspectos Psicobiológicos actualizados, que interesen más a los estudiantes.

- Con el objetivo de evitar sobrecarga de trabajo en casa por parte de los estudiantes (se quejaron durante las sesiones de genética del escaso tiempo disponible), podría subir con antelación los documentos a la plataforma EV, de manera que ellos tengas más tiempo para reflexionar sobre los mismos.

- No todos los alumnos que acudían a clase realizaron el portafolio. El año próximo, incrementaré el valor del porcentaje de peso sobre el total de la asignatura (de 5\% lo incrementaré al 15\%) y pediré que sea obligatorio para los alumnos que quieran realizar evaluación continua de la asignatura.

- También sería interesante hacer más partícipe al alumnado promoviendo que sean ellos los que propongan casos tratados en los debates o cuestiones de grupo grande (de forma que se trabajen conceptos globales con sus ejemplos específicos), ya sean casos inventados o creados (escritos, vídeos...).

- Sería necesario extrapolar este tipo de enseñanza a las clases de otras asignaturas de Psicobiología, para que los alumnos puedan continuar avanzando en este modelo de aprendizaje. 


\section{Conclusiones}

Tras haber aplicado un ciclo de mejora completo en una asignatura de Psicobiología, y tras evaluar los resultados obtenidos, he de destacar lo positivo de este proceso continuo de aprendizaje no sólo para los estudiantes sino también para la docente.

Los resultados que se han ido obteniendo con el portfolio de los estudiantes, tras el desarrollo de la secuencia de actividades y la actitud de los estudiantes durante el transcurso de las sesiones, han mostrado un acierto importante en la metodología docente aplicada, resultando sesiones más dinámicas y amenas que en cursos anteriores.

Además, el resultado obtenido tras el análisis de las escaleras de aprendizaje resulta motivante para continuar desarrollando y mejorando el modelo metodológico propuesto en este curso académico.

Por último, la transferencia y extrapolación de conocimiento de esta asignatura a otras de Psicología sería idóneo, por lo que podría ser una idea de futuro realizar CIMAs de asignaturas integradas en Psicología, tal y como se ha realizado en otros Grados de nuestra Universidad.

Jornadas de Formación e Innovación Docente del Profesorado | № 2 (2019) Esta obra se distribuye con la licencia Creative Commons Reconocimiento-NoComercial-SinObraDerivada 


\section{Referencias bibliográficas}

Bain, K. (2007). Lo que hacen los mejores profesores universitarios. Valencia: Publiciones Universidad de Valencia.

Finkel, D. (2008). Dar clases con la boca cerrada. Valencia: Publicaciones Universidad de Valencia.

Martín-Monzón, Isabel María (2017). Innovando en docencia universitaria. Incorporación de metodología participativa y aprendizaje basado en problemas en asignaturas de Psicobiología. En R. Porlán y E. Navarro-Medina (Coords.), IV Jornadas de Docencia Universitaria (págs. 530-539). Sevilla: Instituto de Ciencias de la Educacion de la Universidad de Sevilla.

Martín-Monzón, Isabel María (2018). Innovación docente en la Asignatura "Fundamentos de Psicobiología" del Grado en Psicología. En R. Porlán y E. Navarro-Medina (Coords.), V Jornadas de Docencia Universitaria (págs. 882-897). Sevilla: Instituto de Ciencias de la Educacion de la Universidad de Sevilla

Mora, F. (2017). Neuroeducación: Solo se puede aprender aquello que se ama. Madrid: Alianza Editorial.

Porlán, R. (2017). Enseñanza Universitaria, Cómo mejorarla. Madrid: Editorial Morata.

Jornadas de Formación e Innovación Docente del Profesorado | № 2 (2019) Esta obra se distribuye con la licencia Creative Commons 